\title{
Mini-Trampolim
}

Artigo Original

\section{Influência aguda de uma aula de mini-trampolim no agachamento}

\section{Adriana Lemos'}

adrianalemos@superig.com.br

\section{Roberto Simão}

robertosimao@ig.com.br

\section{Fabrício Miranda'}

fab.miranda@zipmail.com.br

\section{Jefferson Novaes ${ }^{1,2}$}

jnovaes@terra.com.br

\author{
'Mestrado em Ciência da Motricidade Humana - Universidade Castelo Branco- R.J \\ (LABIMH/ PROCIMH) \\ ${ }^{2}$ Escola de Educação Física e Desportos - Universidade Federal do Rio de Janeiro \\ (EEFD/UFRJ)
}

Lemos A, Simão R, Miranda F, Novaes J. Influência aguda de uma aula de mini-trampolim no agachamento. Fit Perf J.2007;6(2):76-81.

RESUMO - As aulas realizadas no mini-trampolim representam mais uma das opções de treino aeróbico oferecidas nas academias com capacidade de promover melhora no condicionamento físico geral. $\bigcirc$ treino combinado de aeróbica e força tem gerado muitas discussões sobre o efeito deletério que o primeiro pode produzir no segundo, quando praticado de forma subseqüente. Sendo assim, o objetivo deste estudo foi verificar a influência aguda de uma aula de 40 minutos no mini-trampolim sobre o número de repetições alcançados no agachamento. Participaram 12 mulheres praticantes de ambas as atividades (força e mini-trampolim) há pelo menos seis meses. $\bigcirc$ estudo envolveu quatro dias de testes com intervalo de 48 horas entre os mesmos. No primeiro dia foi realizado um teste de $10 R M$ no exercício de agachamento. No segundo dia foi realizado um re-teste para verificar a reprodutibilidade das cargas. $\bigcirc$ terceiro dia envolveu a aula no mini-trampolim e em seguida o exercício de agachamento com as cargas pré-determinadas. No quarto dia, as voluntárias realizaram somente o exercício de agachamento com as cargas obtidas para 10RM. Na análise dos dados foi utilizada ANOVA de duas entradas com medidas repetidas, seguida do teste post-hoc de Tukey $(p<0,05)$. Após os testes realizados, verificou-se que a aula no mini-trampolim provocou significativo decréscimo do número de repetições no exercício agachamento, quando comparado ao desempenho deste exercício sem realização prévia da aula de mini-trampolim. Pode-se concluir que a aula no mini-trampolim traz como efeito agudo a influência negativa no desempenho da força em mulheres treinadas.

Palavras-chave: Treinamento de força, treinamento aeróbico, fadiga muscular, exercícios resistidos, treinamento concorrente.

\section{Endereço para correspondência:}

Rua Enfermeira Ana Maria Barbosa de Almeida, 943/103 Jardim Cidade Universitária, João Pessoa/PB CEP: 58052-270

Data de Recebimento: Julho / 2006

Data de Aprovação: Novembro / 2006

CopyrightC 2007 por Colégio Brasileiro de Atividade Física Saúde e Esporte. 
Acute influence of a mini-trampolim class on squat

The mini-trampolim classes represent one more aerobic training option in gyms and improve the fitness level. The combination between aerobic and strength performance suggests evidences that endurance training, if applied before resistive training $(\mathrm{RE})$, may influence the performance of the last one. So, this study intends to verify the acute influence of a 40 minutes mini-trampolim class over the number of repetitions in an RE. The sample we have used included 12 women, who have practiced both activities (RE and mini-trampolim) for at least six months. The study has included four visits, with a 48-hour interval between them. In the first visit we have evaluated the maximum weight in 10 maximum repetitions (10RM). In the second day the 10RM re-test was made. In the third day, the individuals were submitted to mini-trampolim session and then the squat was made with loads determined in 10RM. In the fourth day, the volunteers get only the squat with loads obtained in 10RM. In order to verify the behavior of the number of repetitions, we have used a two-entry factorial ANOVA, followed by the post-hoc Tuckey $(p<0.05)$ test. When we compared the number of RE repetitions, without previous mini-trampolim class and after a mini-trampolim class, all the sets compared have shown a reduction in the number of repetitions. We have verified that the mini-trampolim class has a negative influence on RE performance in trained women.

Keywords: Strength training, aerobic training, muscular fatigue, resistive exercises, concurrent training.
Influencia aguda de una clase de mini-trampolín en la agachada

Las clases realizadas en el mini-trampolín representan más una de las opciones de entrenamiento aeróbico ofrecidas en las academias con capacidad de promover mejora en el condicionamiento físico general. El entrenamiento combinado de aeróbica y fuerza están generando muchas discusiones sobre el efecto deletéreo que el primero puede producir en el según, cuando practicado de forma subsiguiente. Siendo así, el objetivo de este estudio fue a verificar la influencia aguda de una clase de 40 minutos en el mini-trampolín sobre el número de repeticiones alcanzados en la agachada. Participaron 12 mujeres practicantes de ambas las actividades (fuerza y mini-trampolín) hay al menos seis meses. El estudio envolvió cuatro días de pruebas con intervalo de 48 horas entre los mismos. En el primer día fue realizada una prueba de 10RM en el ejercicio de agachada. En el segundo día fue realizado uno repaso para verificar la reproducibilidad de las cargas. El tercer día envolvió la clase en el mini-trampolín y luego el ejercicio de agachada con las cargas pre-determinadas. En el cuarto día, las voluntarias realizaron solamente el ejercicio de agachada con las cargas obtenidas para 10RM. En el análisis de los dados fue utilizada ANOVA de dos entradas con medidas repetidas, seguida de la prueba post-hoc de Tukey $(p<0,05)$. Tras las pruebas realizadas, se verificó que la clase en el mini-trampolín provocó significativo decrecimiento del número de repeticiones en el ejercicio agachada, cuando comparado al desempeño de este ejercicio sin realización previa de la clase de mini-trampolín. Se puede concluir que la clase en el mini-trampolín trae como efecto agudo la influencia negativa en el desempeño de la fuerza en mujeres entrenadas.

Palabras-claves: Entrenamiento de fuerza, entrenamiento aeróbico, fatiga muscular, ejercicios resistidos, entrenamiento competidor.

\section{INTRODUÇÃO}

A investigação sobre a interação entre dois diferentes componentes da aptidão física, aeróbico e força, tem demonstrado em algumas, mas não em todas as ocasiões, que o desenvolvimento da força ou resistência pode ser afetado pelo treinamento concorrente $^{15}$. Alguns autores ${ }^{11,19}$ argumentam que a realização de um treinamento combinado envolvendo o trabalho em exercícios resistidos (ER) e aeróbico acarreta melhores ganhos de força em comparação com indivíduos que realizam somente treinamento de força. No entanto, outros estudos ${ }^{5,17,20}$ revelam que ainda há uma grande indefinição sobre os reais efeitos fisiológicos, morfológicos e neuromusculares decorrentes desse tipo de treinamento.

Tais controvérsias verificadas nos estudos acima podem ocorrer em função de vários fatores, como por exemplo, o nível de condicionamento dos indivíduos, o volume e a freqüência de treinamento, além da experiência motora e da forma como os dois métodos de treinamento são integrados.

Nas academias, muitas atividades são oferecidas com o propósito de desenvolver melhor aptidão aeróbica. Dentre elas, podemos destacar o mini-trampolim, que consiste numa aula que permite a realização de exercícios que envolvem força da gravidade além da aceleração e desaceleração. Isso é possível, devido a sua superfície elástica e sistema de fixação de molas, de especial resistência, que permitem atingir alto desempenho na execução dos exercícios.

As aulas no mini-trampolim podem ser pré-coreografadas ou não, mas costumam ser constituídas de exercícios ritmados de membros superiores e inferiores de forma combinada. Seus benefícios são basicamente os mesmos que os alcançados pela prática regular de exercícios aeróbicos ${ }^{9,14}$. Portanto, quando esta atividade for combinada com os ER, pode-se questionar se haverá alguma interferência no desempenho deste último.

Sendo assim, o objetivo deste trabalho é verificar a influência aguda de uma aula de 40 minutos no mini-trampolim sobre o número de repetições em três séries de agachamento com cargas para 10 repetições máximas (10RM), em mulheres treinadas, e comparar com o número de repetições alcançados no mesmo exercício sem a execução prévia do mini-trampolim.

\section{MATERIAIS E MÉTODOS}

\section{Amostra}

Participaram da amostra 12 mulheres voluntárias $(25 \pm 6$ anos; $164 \pm 6 \mathrm{~cm} ; 55,6 \pm 4,6 \mathrm{~kg}$ ). Todas referiram ser saudáveis e 
fisicamente ativas, realizando atividades aeróbicas e de força pelo menos quatro vezes por semana e familiarizadas com os exercícios e equipamentos utilizados neste estudo. Para seleção da amostra respeitaram-se os seguintes critérios de inclusão:

a) prática regular de atividades aeróbicas e de força há pelo menos seis meses;

b) IMC $\leq 25 \mathrm{Kg} \cdot \mathrm{m}-2$, evitando-se níveis de sobrepeso que comprometessem a qualidade de execução dos movimentos;

c) questionário PAR-Q negativo.

Todas as voluntárias assinaram um termo de consentimento, de acordo com a Resolução 196/96 do Conselho Nacional de Saúde do Brasil.

Adotaram-se como critérios de exclusão:

a) uso de medicamentos que tivessem influência sobre o comportamento das respostas funcionais, principalmente sobre a freqüência cardíaca;

b) problemas osteomioarticulares que poderiam limitar a realização dos exercícios propostos;

c) falta de familiarização com a aula no mini-trampolim e no exercício agachamento no aparelho Smith.

\section{Teste de 10RM}

Os dados foram coletados em dois dias distintos. Na primeira visita, os indivíduos, após realizarem as medidas antropométricas, executaram o teste de 10RM no aparelho de agachamento Smith (Fitness equipment; Franklin Park, IL).

Após a obtenção das cargas máximas no teste de 10RM, os indivíduos descansaram por 48 horas e foram reavaliados para obtenção da reprodutibilidade das cargas no teste de 10RM (segundo dia de teste). Para tanto, considerou-se como 10RM a maior carga estabelecida em ambos os dias, com diferença menor que $5 \%$. Nos intervalos entre as sessões de testes não foi permitida a realização de exercícios para membros inferiores, a fim de evitar qualquer interferência nos resultados obtidos.

Visando reduzir a margem de erro nos testes de 10RM, foram adotadas as seguintes estratégias:

a) instruções padronizadas foram fornecidas antes do teste, de modo que o avaliado estivesse ciente de toda a rotina que envolvia a coleta de dados;

b) o avaliado foi instruído sobre a técnica de execução do exercício;

c) o avaliador estava atento quanto à posição adotada pelo praticante no momento da medida, pois pequenas variações no posicionamento das articulações envolvidas no movimento poderiam acionar outros músculos, levando a interpretações errôneas dos escores obtidos;

d) estímulos verbais foram realizados a fim de manter alto o nível de estimulação; e) os pesos adicionais utilizados no estudo foram previamente aferidos em balança de precisão.

Os intervalos entre as tentativas em cada exercício durante o teste de 10RM foram fixados entre 2 e 5 minutos $^{4}$.

\section{Procedimento para coleta de dados}

Na terceira e quarta visitas, após 48 horas da obtenção das cargas nos testes de 10RM, os indivíduos foram submetidos a duas sessões de treinamento, com intervalo de 48 horas entre as mesmas. Nos intervalos entre as sessões, não foi permitida a realização de exercícios que pudessem influenciar nos dados.

A condução do treinamento durante a terceira visita consistia na realização do mini-trampolim (40 minutos) e posteriormente a realização de três séries de agachamento com cargas de 10RM até a falha concêntrica. Os intervalos entre as séries, bem como o período compreendido entre o fim da aula de mini-trampolim e o início do exercício, foram fixados em 2 minutos. A capacidade de desempenho observada através do número de repetições foi determinada pela exaustão ou incapacidade de manter o padrão do movimento completo.

A quarta visita consistiu em um aquecimento específico, em duas séries, com intervalo de 1 minuto entre as séries, em 15 repetições com $40 \%$ da carga de 10RM. A capacidade de desempenho nas três séries do exercício de agachamento seguiram o mesmo procedimento da terceira visita, exceto que não havia a realização prévia da aula do mini-trampolim. A inclusão dos indivíduos na terceira e quarta visitas nas seqüências foram determinadas na forma de delineamento alternado.

\section{Características da aula no mini-trampolim}

A aula foi composta por três fases:

a) 5 minutos de aquecimento no solo;

b) 30 minutos da fase aeróbica com ritmo de 145 bpm, sobre o mini-trampolim, com movimentos de polichinelo, elevação de joelhos e calcanhares, corrida e chute combinados com movimentos dos braços;

c) 5 minutos de resfriamento no solo.

Todos os exercícios propostos foram apresentados em forma de coreografias simples e de fácil execução.

\section{Tratamento estatístico}

Para o tratamento dos dados utilizou-se uma ANOVA de duas entradas (seqüência $x$ número de séries) com medidas repetidas no segundo fator em associação ao teste post-hoc de Tukey. A determinação da reprodutibilidade nas cargas para 10RM foi realizada através do coeficiente de correlação intraclasse, adicionada ao teste $t$ de student pareado. $\bigcirc$ nível de significância adotado foi de $5 \%$ e os dados foram tratados no software Statistica 5.5 (Statsoft $\AA$, USA). 
Inicialmente são ilustrados os resultados referentes à reprodutibilidade das cargas obtidas nos testes de 10RM.

Como pode ser constatado, foram verificados elevados valores coeficientes de correlação intraclasse no exercício selecionado $(r=0,94)$. Em adição, o teste-t student pareado não demonstrou diferença significativa entre as cargas obtidas para 10RM no exercício de agachamento $(p>0,05)$. Ao comparar o número de repetições intra-seqüência no exercício agachamento após a sessão de mini-trampolim, observou-se uma redução significativa em todas as séries desempenhadas (Figura 1).

Foram verificadas diferenças significativas entre a $1^{a}$ e a $3^{a}$ série $(p=0,004)$, entre a $1^{a}$ e a $2^{a}(p=0,0008)$ e entre a $2^{a}$ e a $3^{a}$ $(p=0,02)$. Já no dia em que o agachamento foi realizado sem prévia sessão do mini-trampolim, foram observadas reduções significativas no desempenho intra-sequência apenas entre a $1^{a}$ e $3^{a}$ série (Figura 1).

Ao comparar o número de repetições entre as séries (intersequência) sem execução prévia do mini-trampolim, e com a execução após sessão do mini-trampolim, observou-se uma redução significativa do número de repetições em todas as séries desempenhadas (Figura 2).

\section{DIscussão}

No presente estudo verificou-se que uma sessão de 40 minutos de mini-trampolim influenciou negativamente nas três séries de agachamento realizadas subseqüentemente.

Observou-se uma redução significativa no número de repetições em todas as três séries, quando comparadas à mesma seqüência, sem a prévia realização da sessão no mini-trampolim (Figura 2). Através da análise da intra-sequência, verificou-se uma redução significativa em todas as séries desempenhadas após a sessão de mini-trampolim.

mesmo não aconteceu no dia em que o agachamento foi realizado sem a sessão prévia de mini-trampolim, quando foram observadas reduções significativas no desempenho intra-sequência apenas entre a $1^{a}$ e $3^{a}$ série (Figura 1). Sendo assim, os resultados aqui apresentados parecem concordar com diversos estudos que utilizaram outras modalidades de exercícios aeróbicos prévios, e que demonstraram a influência negativa nos $\mathrm{ER}^{3,12}$.

Tal modalidade, a aula no mini-trampolim, foi avaliada em laboratório por Furtado et al. ${ }^{9}$, que verificaram o comportamento das variáveis funcionais em 10 mulheres treinadas na modalidade de Jump Fit (mini-trampolim), e verificaram que a intensidade média da aula correspondeu a $75 \%( \pm 7,7)$ do $\mathrm{VO}_{2 \text { ico }}$. A avaliação espirométrica das diversas etapas da aula revelou os seguintes resultados médios: $\mathrm{FC}$ de 160,3 b.p.m $( \pm 8,9), \mathrm{VO}_{2}$ de 1,59 L.min- $1( \pm 0,45), Q R 0,87( \pm 0,10)$ e dispêndio energético total $386,4 \mathrm{kcal}( \pm 13,8)$.
Conclui-se que a partir da magnitude das respostas funcionais, a aula do Jump Fit proporciona aumento da resistência cardiorespiratória, contribuindo de forma efetiva para a manutenção e melhora da aptidão física e saúde na qualidade de vida. Em que pesem diferenças metodológicas entre ambas as modalidades de aula, uma sessão de mini-trampolim pode ser caracterizada como uma atividade de moderada a alta intensidade, devido a sua similaridade de execução ao Jump Fit.

Sendo assim, pode-se supor que devido à intensidade do esforço solicitado no mini-trampolim, a influência da fadiga foi sentida no momento da realização das três séries no agachamento.

Estudos demonstram que a influência dos exercícios aeróbicos antes do treinamento de força possui uma duração média de 21 a 160 minutos $^{3}$. Em um estudo proposto por Lemos et al. ${ }^{14}$, os autores observaram evidências de que uma sessão de exercícios aeróbicos antecedendo os ER pode influenciar no desempenho deste último.

objetivo deste estudo foi verificar a influência de uma aula de Jump Fit sobre o número de repetições em uma seqüência nos ER. A amostra foi de sete mulheres praticantes de ER e Jump Fit. $\bigcirc$ estudo envolveu quatro visitas com intervalo de 48 horas entre as mesmas.

Na primeira visita, foram realizados os testes de $10 R M$ e, após 48 horas, realizaram-se um re-teste de 10RM. Nos dois dias seguintes, após a obtenção das cargas, os indivíduos foram submetidos a duas sessões de treinamento, organizadas em duas formas seqüenciais (SEQ A e SEQ B): SEQA - leg press 450 , cadeira extensora e cadeira flexora, sendo três séries com cargas de 1ORM, e intervalos de 2 minutos entre as séries e exercícios. A SEQB - consistia primeiramente na aula de Jump Fit e, em seguida, a realização dos ER na mesma ordenação da SEQA.

A realização das seqüências foi definida pelo balance cross-over design. Comparando-se o número total de repetições dos ER sem a execução prévia do Jump Fit e após o Jump Fit, verifica-se que todas as séries em questão apresentaram redução no número de repetições, com exceção da $1^{a}$ série da flexão do joelho. Dessa forma, os autores verificaram que a aula de Jump Fit influencia negativamente o desempenho dos ER.

Em que pesem similaridades metodológicas entre os estudos, algumas diferenças são claras. Utilizamos o exercício de agachamento, que envolve uma maior massa muscular e também um maior grau de dificuldade de execução do que o leg- press. Também observarmos nos resultados de Lemos et al. ${ }^{14}$, que na primeira e segunda série no exercício leg-press, a redução no número de repetições foi pouco evidenciada (9,5 repetições). Já no presente estudo, observou-se a redução pronunciada do número de repetições na primeira série de agachamento após a aula de mini-trampolim (7,2 repetições).

Talvez tal diferença realmente possa ser associada à massa muscular envolvida no exercício, e ao grau de dificuldade na técnica de execução do movimento. Ou seja, numa aula de minitrampolim, a fadiga pode ser mais expressiva em um exercício 
FigURA 1

NúMERO de REPETIÇões INTRA-SEQÜÊNCIA NO EXERCíCIO AGACHAMENTO APÓS A SESSÃO DE MINI-TRAMPOLIM E SEM PRÉVIA SESSÃO DO MINI-TRAMPOLIM
Figura 2

NúMERo de REPETIÇÕes ENTRE AS SÉrIES SEM EXECUÇÃo PRÉVIA do MINI-TRAMPOLIM, COM A EXECUÇÃO APÓS SESSÃO DO MINI-TRAMPOLIM

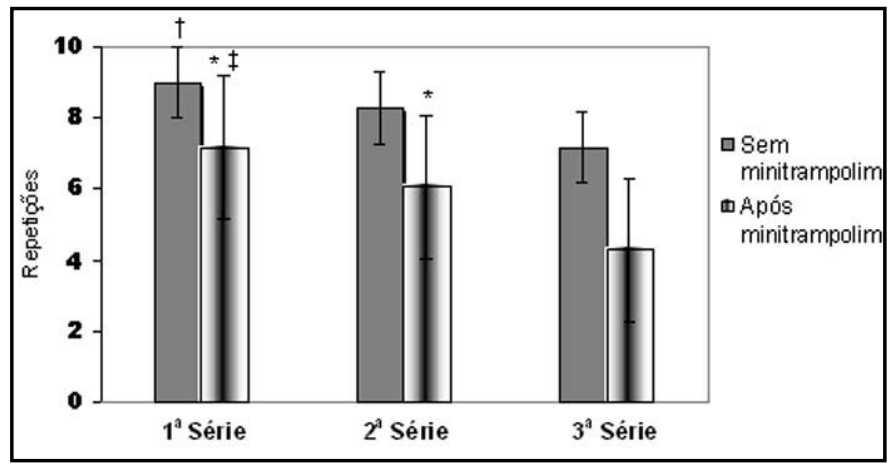

† Diferença significativa em relação à $3^{a}$ série

* Diferença significativa em relação à $3^{a}$ série

‡Diferença em relação à $2^{a}$ série

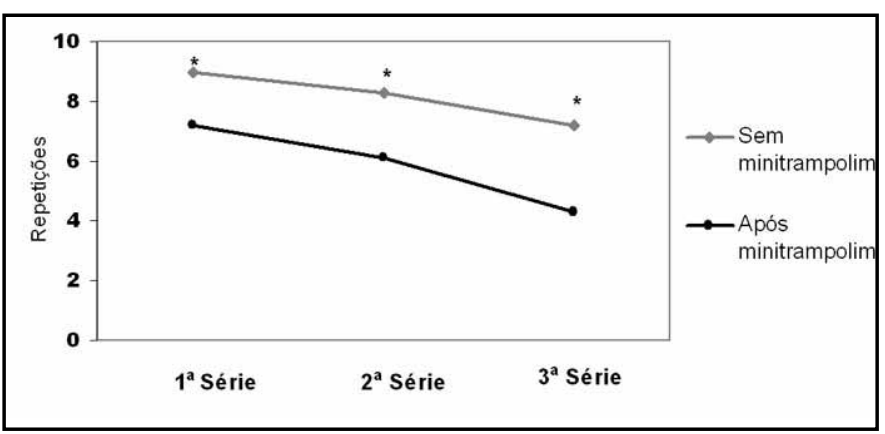

Observou-se uma redução significativa do número de repetições em todas as séries comparadas.

* Diferenças entre as séries comparadas $(p<0,05)$

de metabólitos (isto é, fosfato inorgânico, ácido lático, amônia) e esgotamento de substratos de energia, como ATP, fosfato de creatina e glicogênio muscular ${ }^{16}$.

Tem sido sugerido que o decréscimo do $\mathrm{pH}$ no músculo como resultado de acúmulo de lactato seja uma causa fundamental da fadiga em exercícios de curta duração. Níveis elevados de lactato no sangue são evidentes após o exercício de resistência de alta intensidade ${ }^{8}$. $O$ lactato do sangue normalmente retorna aos níveis de repouso aproximadamente uma hora após o término do exercício ${ }^{8}$. A força tem-se mostrado reduzida até quatro horas após o término do exercício de resistência² ${ }^{2}$.

Os fatores influenciadores mais prováveis podem ser: o estresse térmico, a desidratação, o percentual do $\mathrm{VO}_{2 \text { pico }}$ no qual o indivíduo se exercita, o limiar de lactato do indivíduo, a percentagem de fibras do tipo I recrutadas, a biomecânica de execução e o conteúdo de glicogênio muscular ${ }^{3}$.

Para testar a hipótese da depleção dos estoques de glicogênio, Aoki et al. ${ }^{3}$ verificaram se o consumo de carboidrato seria capaz de atenuar os efeitos de uma sessão aguda de exercício de resistência sobre o desempenho da força.

Para tanto, seis universitárias fisicamente ativas, com experiência em treinamento de força, foram submetidas a um teste para determinação do $\mathrm{VO}_{2 \text { pico' }}$ e a um teste de $1 \mathrm{RM}$ para o leg press, seguido de um teste de repetições máximas (duas séries de leg press realizadas a 70\% de 1RM até a exaustão) em dias diferentes.

O procedimento envolvia um protocolo duplo-cego, em que os sujeitos eram submetidos a duas condições experimentais, recebendo uma bebida placebo e outra contendo carboidratos, 
antes e durante a realização de corrida em esteira a $70 \%$ do $\mathrm{VO}_{2 \text { máx }}$. por 45 minutos.

Em seguida ao exercício de resistência, os indivíduos realizaram um teste de 1RM seguido pelo teste de repetições máximas. Seus resultados demonstraram que houve redução do número de repetições nas duas situações, ou seja, independentemente do mecanismo envolvido na redução do número de repetições máximas, o consumo de carboidrato foi incapaz de reverter o efeito agudo causado pela sessão de resistência.

A hipótese associada diretamente ao efeito agudo sugere que o treinamento aeróbico possivelmente interfira na capacidade do sistema neuromuscular em gerar a força máxima ${ }^{1,19}$. Embora um número considerável de estudos tenha relacionado as adaptações no desempenho com o treinamento concorrente, poucos analisaram os mecanismos fisiológicos marcantes responsáveis pela inibição da força ${ }^{3,12}$.

A grande limitação metodológica do presente estudo recai sobre a falta de outro determinante de fadiga além do utilizado, que foi o número de repetições. Sugerimos que, em estudos futuros, a utilização do lactato possa ser empregada em associação à redução do número de repetições.

\section{CONCLUSÃO}

Com base nos resultados encontrados no presente estudo, pode-se concluir que a aula no mini-trampolim apresenta alta intensidade e volume de trabalho, trazendo como efeito agudo a influência negativa no desempenho da força em mulheres treinadas, quando realizada antes do treino de força.

Contudo, quando o objetivo principal do treinamento recair na melhoria do condicionamento cardiorespiratório, o treinamento no mini-trampolim parece ser uma boa opção de treino nas academias. Cabe também destacar, que a magnitude da influência das aulas de mini-trampolim no desempenho agudo da força tende a ser mais pronunciada com a evolução das séries.

Nesse sentido, uma sessão de exercícios realizados com séries simples pode não exercer tanta influência negativa no desempenho da força.

Estudos futuros necessitam ser conduzidos para melhor investigar a influência da aula de mini-trampolim sobre o desempenho do treino de força.

\section{REFERÊNCIAS BIBLIOGRÁFICAS}

1. ABERNETHY, PJ; QUIGLEY, BM. Concurrent strength and endurance training of the elbow extensors. Journal of Strength and Conditioning Research. 1993; 7: 234-240.

2. ABERNETHY, PJ. Influence of acute endurance activity on isokinetic strength. Journal of
Strength and Conditioning Research. 1993; 7: 141-145.

3. AOKI, MS; JUNIOR, LP; NAVARRO, F; UCHIDA, MC; BACURAU, RF. Suplementação de carboidrato não reverte o efeito deletério do exercício de endurance sobre o subseqüente desempenho de força. Revista Brasileira de Medicina do Esporte. 2003; 9: 282-287.

4. BAECHLE, TR; EARLE, RW. Essentials of strength training and conditioning. Champaign: Human Kinetics, 2000

5. BELL, GJ; PETERSEN, B; WESSEL, J; BAGNALL; QUINNEY, HA. Physiological adaptations to concurrent endurance training and low velocity resistance training. International Journal Sports Medicine. 1991; 12: 384-390.

6. CRAIG, BW; LUCAS, J; POHLMAN, R; STELLING, H. Effects of running weightlifting and a combination of both on growth hormone release. Journal Apply Sport Science Research. 1991; 5: 198-203.

7. DUDLEY, GA; DJAMIL, R. Incompatibility of endurance and strength training modes of exercises. Journal Apply Physiology. 1985; 59: 1446-1451.

8. FOXDAL, P; SJODIN, A; SJODIN, B. Comparison of blood lactate concentrations obtained during incremental and constant intensity exercise. International Journal Sports Medicine. 1996; 17: 360-365

9. FURTADO, ES; SIMÃO, R; LEMOS, A. Análise do consumo de oxigênio, freqüência cardíaca e dispêndio energético, durante as aulas do Jump Fit. Revista Brasileira de Medicina do Esporte. 2004; 10: 371-375.

10. GOMES, RV; MATSUDO, SMM; ALEMIDA, VCS; AOKI, MS. Suplementação de carboidrato associada ao exercício de força não afeta o subseqüente desempenho no teste de potência aeróbia. Revista Brasileira de Ciência e Movimento. 2003; 1: 67-72.

11. GRAVELLE, BL; BLESSING, DL. Physiological adaptation in women concurrently training for strength and endurance. Journal of Strength and Conditioning Research. 2000; 14: 5-13

12. HICKSON, RC; DVORAK, BA; GOROSTIGA, EM; KUROWISKI, TT; FOSTER, C. Potential for strength and endurance training to amplify endurance performance. Journal Apply Physiology. 1988; 65: 2285-2290.

13. KRAEMER, WJ; PATTON, JF; GORDON, SE; HARMAN, EA; DESCHENES, MR; REYNOLDS, $\mathrm{K}$, et al. Compatibility of high-intensity strength and endurance training on hormonal and skeletal muscle adaptations. Journal Apply Physiology. 1995; 78: 976-89.

14. LEMOS, A; SIMÃO, R; MIRANDA, H; NOVAES, J. A influência de uma sessão de Jump Fit no desempenho dos exercícios resistidos. Revista Brasileira de Fisiologia do Exercício. Aceito para publicação, 2005.

15. LEVERITT, M; ABERNETHY, PJ; BARRY, B; LOGAN, P. Concurrent strength and endurance training: The influence of dependent variable selection. Journal of Strength and Conditioning Research. 2003; 17: 503-508.

16. LEVERITT, M; ABERNETHY, PJ. Acute effects of high intensity endurance exercise on subsequent resistance activity. Journal of Strength and Conditioning Research. 1999; 3: 47-51.

17. LEVERITT, M; ABERNETHY, PJ. Concurrent strength and endurance. Sports Medicine. 1999; 28: 413-427.

18. LEVERITT, M; MACLAUGHLIN; ABERNETHY, PJ. Changes in leg strength 8 and 32 hours after endurance exercise. Journal Sport Science. 2000; 18: 865-879.

19. MCCARTHY, JP; AGRE, JC; GRAF, BK; POZNIAK, MA; VAILAS, AC. Compatibility of adaptative responses with combining strength and endurance training. Medicine Science Sports Exercise. 1995; 27: 429-436.

20. NELSON, AG; ARNEL,L DA; LOY, SF; SILVESTER, LJ; CONLEE, RK. Consequences of combining strength and endurance training regimes. Physical Therapy. 1990; 70: 287-294 